\title{
From erectile dysfunction to brain subependymoma: A case report.
}

\section{Authors: Tatjana Bacun ${ }^{1,2}$, Aleksandar Kibel ${ }^{1,3}$, Dunja Degmecic ${ }^{4,5}$, Roman Pavic ${ }^{6,7}$}

A $1{ }^{1}$ Internal Medicine Clinic, University Hospital Centre Osijek, Osijek, Croatia

Vepartment of Internal Medicine, Faculty of Medicine, University of Osijek, Croatia

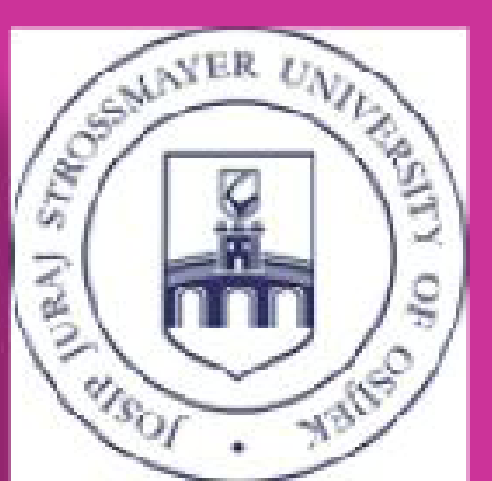

${ }^{3}$ Department of Physiology and Immunology, Faculty of Medicine, University of Osijek, Croatia

${ }^{4}$ Clinic of Psychiatry, University Hospital Centre Osijek, Osijek, Croatia

${ }^{5}$ Department of Psychiatry, Faculty of Medicine, University of Osijek, Croatia

${ }^{6}$ University Hospital of Traumatology, Zagreb, Croatia

${ }^{7}$ Department of Surgery, Faculty of Medicine, University of Osijek, Osijek, Croatia
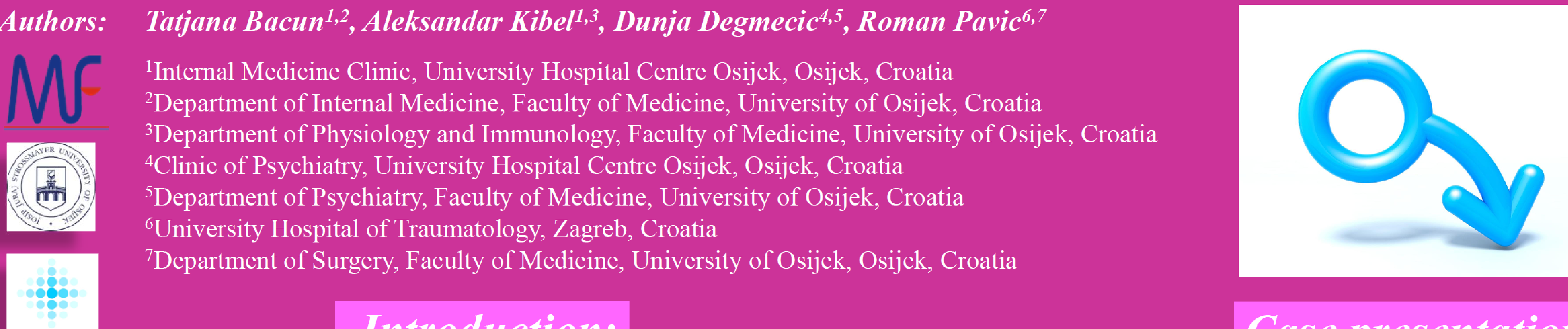

\section{Introduction:}

Case presentation:

Endocrinopathies are rare causes of erectile dysfunction and previously cases have been reported where erectile dysfunction (as a main presenting feature) was caused by hyperprolactinemia due to a pituitary adenoma [1, 2].

In this case report we present a patient with erectile dysfunction, where at first, exogenous factors were blamed to be a cause. Only when he was referred to a neurologist because of headaches (and later to an endocrinologist), an underlying brain subependymoma was found.

$\rightarrow 27$-year old male, married for 4 years with no children, presented with possible infertility and erectile dysfunction which was previously interpreted as caused by a poor social and psychological situation in the family.

$\rightarrow$ The patient experienced occasional periorbital and temporal headaches, loss of apetite, general weakness.

$\rightarrow$ endocrinological (mildly elevated prolactin levels and reduced levels of testosterone) and radiological workup (see below).

$\rightarrow$ The patient underwent a left-sided supraorbital craniotomy and complete surgical removal of the tumor at the Dept. of Neurosurgery. Histopathology $\rightarrow$ subependymoma (G II).

$\rightarrow$ One year after surgery, the patient was in good general condition, but with bitemporal hemianopia, with atrophy of the right optic nerve and complete loss of vision in his right eye, panhypopituitarism and impotence.

$\rightarrow$ Two years later, with hormone replacement therapy, there was no sexual dysfunction.

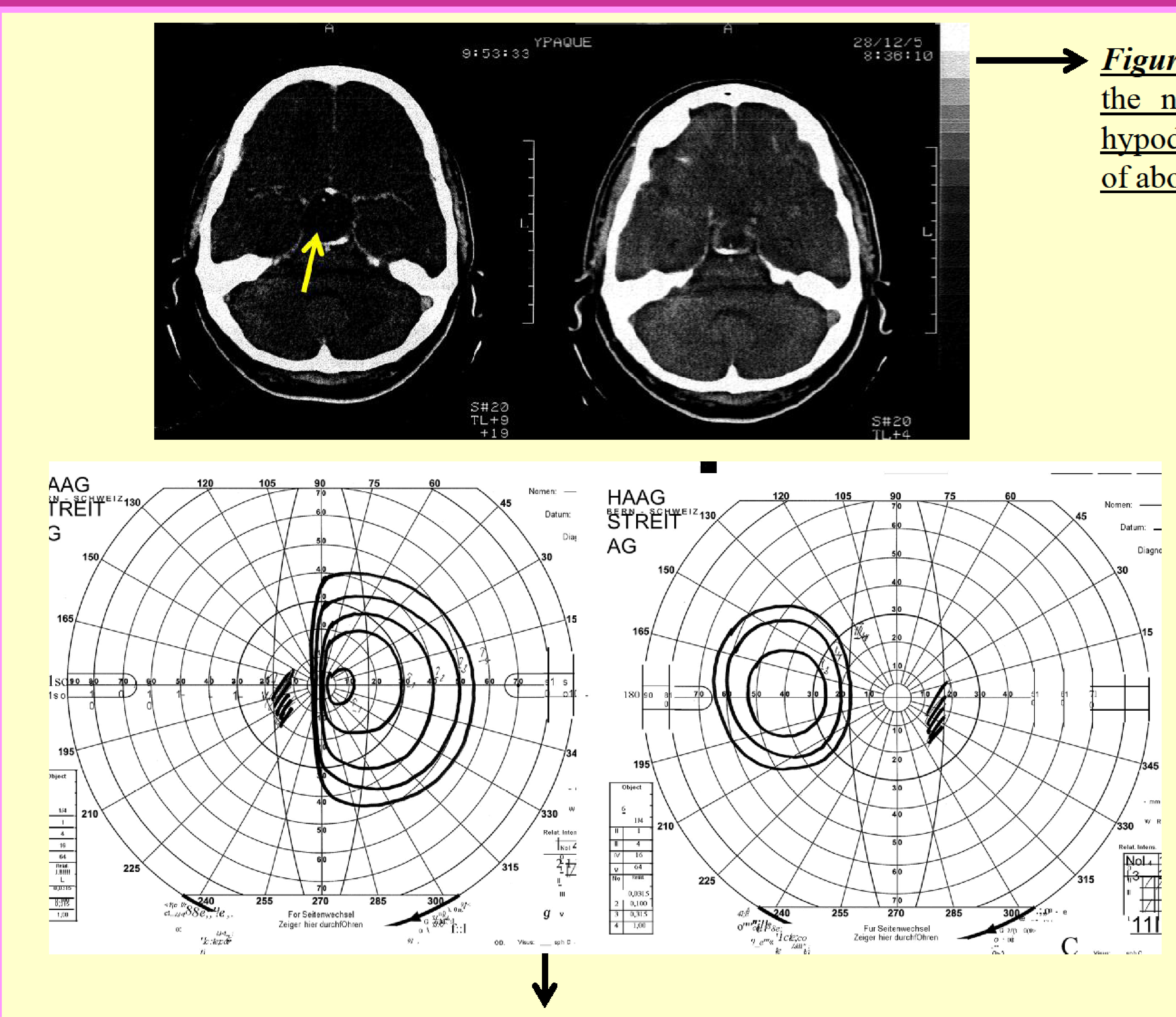

Figure 3. Visual field test (Goldman perimetry) indicating bitemporal hemianopia

Figure 1. Skull CT scan before neurosurgical intervention (left side) and after the neurosurgical procedure (right side). The yellow arrow points to the hypodense oval cystic tumor (initially a suspected cyst with a measured diameter of about $2.7 \mathrm{~cm}$ ) in the sellar and suprasellar area

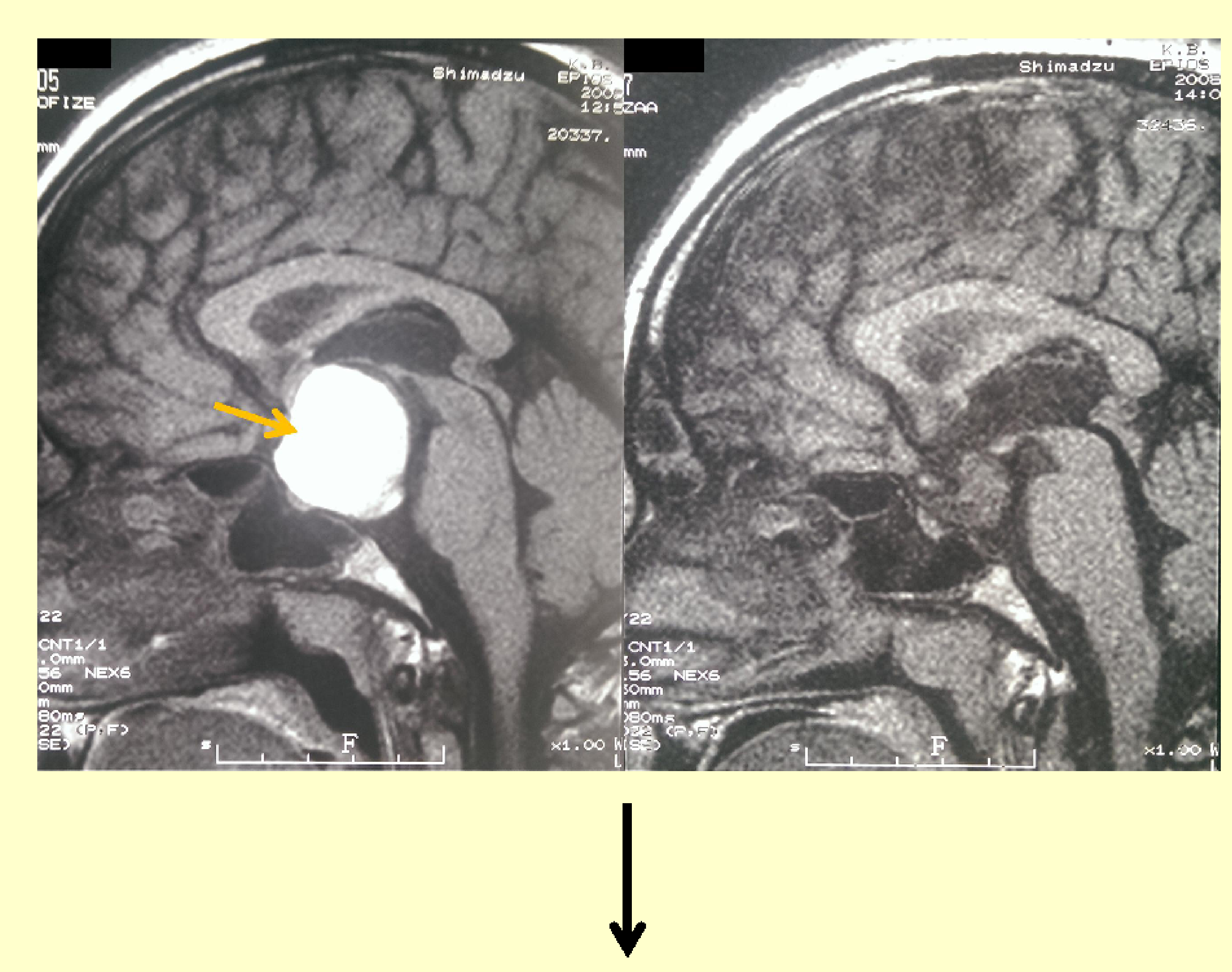

Figure 2. Brain MRI before neurosurgical intervention (left side) and after the neurosurgical procedure (right side). The yellow arrow points to the cystic tumor (with a measured diameter of $3 \mathrm{~cm}$ )

\section{Conclusions:}

The presented case illustrates the need to keep not only endocrinopathies in mind when a patient presents with erectile dysfunction, but also that the causes may be diverse and that rare brain tumors (other than pituitary adenomas) may present themselves with mainly erectile dysfunction. There was an interesting recent case report of a young male with back pain and erectile dysfunction where a spinal myxopapillary ependymoma was determined to be the cause [3], but we are not aware of any previous case reports of brain subependymomas or ependymomas as main causes of erectile dysfunction through endocrine disorders (hypogonadism). In that respect, this important case is extremely interesting and may hopefully be of assistance for clinicians in their everyday work with patients who present with erectile dysfunction as the main problem.

\section{Contact:}

Assist.Professor Tatjana Bacun, MD, PhD

University Hospital Centre Osijek,

J. Huttlera 4, 31000 Osijek, Croatia phone: 00385915135637 e-mail: tbacun@gmail.com

\section{References:}

Anand KS, Dhikav V (2013). Hyperprolactinemia: an unusual cause of erectile dysfunction. Zeitlin SI, Rajfer J (2000). Zeitlin SI, Rajfer J (2000). dysfunction. Reviews in urology 2 : 39-42.

Ngo TP, Dufton J, Stern PJ, Islam O (2013). Myxopapillary ependymoma as a cause of back pain in a young male - A case report. The Journal of the Canadian Chiropractic Association 57: 150-155. 